\title{
Influence of the cutting edge microgeometry on the tool life in austenitic stainless steel machining with carbide end mill
}

\author{
Marius-Bogdan Bozga ${ }^{1 *}$, Marcel Sabin Popa ${ }^{1}$, Stefan Sattel $^{2}$, and Vlad-Bogdan Tomoiagă ${ }^{1}$ \\ ${ }^{1}$ Technical University of Cluj-Napoca Romania, Faculty of Mechanical Engineering, Department of \\ Manufacturing Engineering, Blvd. Muncii, No. 103-105, Cluj-Napoca, Romania \\ ${ }^{2}$ Gühring KG, Head of Research and Development Department, Winterlingen Street, No. 12, 72488, \\ Sigmaringen-Laiz, Germany
}

\begin{abstract}
The goal of this paper is to determine the optimal cutting edge microgeometry for a carbide end mill used in stainless steel machining. The optimal cutting edge microgeometry will be determined through conducting more tool life tests. Tools with three different cutting edge radiuses and one without edge preparation will be tested in order to determine the influence of the cutting edge microgeometry on the wear and tool life. The edge microgeometry is obtained through wet blasting preparation.
\end{abstract}

\section{Introduction}

This study aims to determine the influence of the tools cutting edge microgeometry obtained in the end milling process of a 1.4301 grade austenitic stainless steel through conducting more tool life tests. In order to determine the optimal cutting edge microgeometry, tools with three different cutting edge rounding were prepared through wet blasting with different parameters. The tools were then tested in order to determine de influence of the cutting edge microgeometry obtained through wet blasting on the tool life.

The 1.4301 grade is an austenitic stainless steel, which is widely used in many industries such as automotive industry, food industry, chemical industry, petrochemical industry, decorative items and kitchen utensils for its good corrosion and heat resistance, ease of formability, good cryogenic properties and its aesthetic appearance. This grade of austenitic stainless steel is also known for its poor machinability.

The end milling process is often used in many industries for machining stainless steel parts. The milling process is a hard cutting process due to the interrupted contact between the cutting tool and the work piece and due to the variable chip thickness, therefore an extensive optimisation of the process is necessary [1].

An optimisation of the austenitic stainless steel cutting process is possible through choosing the right tool geometry and cutting parameters [2]. Another efficient way of increasing the tool life is the usage of an efficient cutting fluid, which decreases the cutting

\footnotetext{
* Corresponding author: marius.bozga@yahoo.com
} 
edge temperature, reduces the cutting forces, helps to reduce the friction coefficient between the chip/workpiece and cutting tool, inhibits adhesion and flushes the chips away from the tool and workpiece.

Ciftci and Endrino [2,3] showed that choosing the right coating for the tool also helps to increase the tool life. High temperature hardness, oxidation resistance, low friction coefficient and low thermal conductivity are the main properties of a good coating for a cutting tool used in machining austenitic stainless steel.

The researches regarding cutting edge microgeometry from the last two decades show that the tool life can be increased through preparation of the tools cutting edge with a specific rounding. For each machining process and machined material there is an optimal cutting edge rounding [4].

The present tests were carried out with a Gühring RF100VA solid carbide end mill with four cutting edges and a specific geometry for machining stainless steel. The main purpose of the tool life tests was to determine the influence of the cutting edge microgeometry prepared through wet blasting on the tool life and performance.

\section{Cutting edge microgeometry and preparation}

Currently there are no international standards defining the microgeometry of the cutting edge. The characterisation of a rounded edge defined by a radius is given in DIN 6582, but there are no specific details about the criteria used to measure this radius. Proposals for a classification were made but none of these are universally used [4].There are currently different methods being used for the measuring of the microgeometry and this often leads to significantly different results.

\subsection{Microgeometry classification by form}

The classification of the cutting edge by form is done by analysing the edge profile in a perpendicular section on the cutting edge.

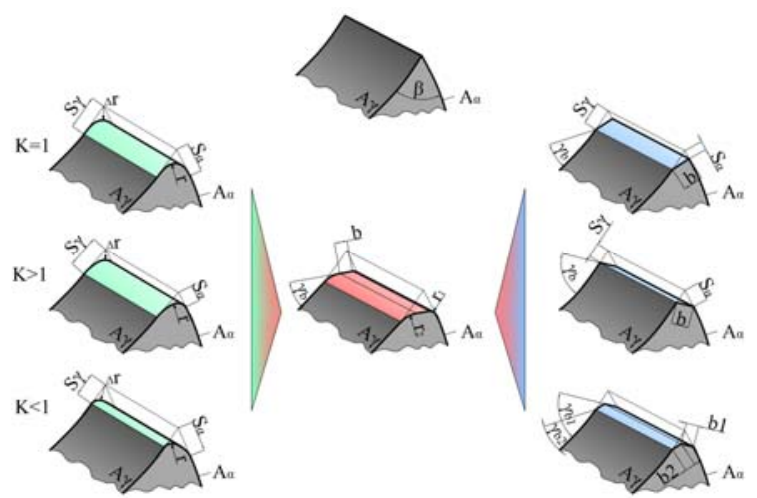

Fig. 1. Typical cutting edge microgeometries.

\subsubsection{Round}

The profile of the perpendicular section to the edge has a round shape defined by a radius $r$ and two lengths $S \gamma$ and $S \alpha . S \gamma$ and $S \alpha$ are the length from the tangent point of the radius with rake face $(S \gamma)$ and flank face $(S \alpha)$ up to the imaginary intersection of the rake face with the flank face. The length $\Delta r$ helps defining the profile flattening [4]. 


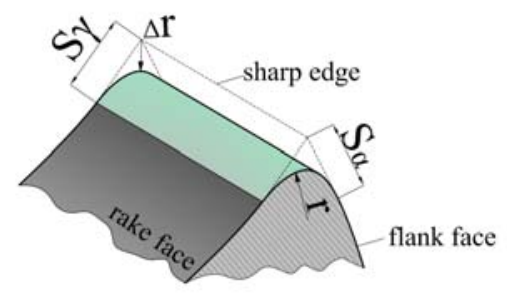

Fig. 2. Characterisation of the round cutting edge.

The $K$-factor is the ratio between $S \gamma$ and $S \alpha$, which shows the orientation of the rounded edge towards the flank face or the rake face [4].
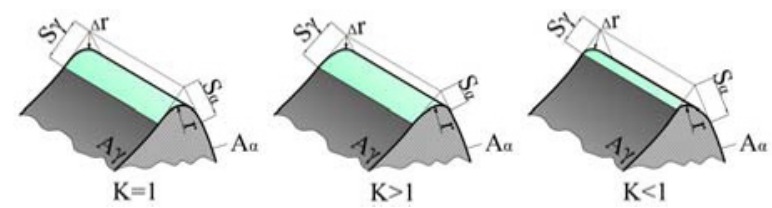

Fig. 3. Different types of cutting edge rounding.

\subsubsection{Chamfered}

It is defined as an intermediary flat surface in between flank and rake face and it is characterised by an angle $\gamma_{b}$ and a width $b$. It can have a single or more flat surfaces and can have a greater inclination towards the flank face or towards the rake face. This inclination is characterised by the $K$-factor.

\subsubsection{Chamfered with rounded edges}

This form is a combination between the rounded and chamfered cutting edge.

\subsection{Microgeometry used in the tests}

In the present tests, tools with cutting edges prepared through wet blasting were used. Wet blasting produced a cutting edge with a round form with a $K$-Factor lower than 1 on the peripheral cutting edge and bigger than 1 on the end cutting edge. The parameters for the wet blasting of the solid carbide end mill were established after intense research and with the help of the specialised personnel from Gühring Company. The interval of the cutting edge rounding values, that were obtained after wet blasting, were chosen after conducting more FEM simulations in order to restrict the interval.

\section{The preparation of the cutting edge microgeometry through wet blasting}

Wet blasting (or Vapormatting) is a process similar to conventional blasting in the way of using abrasive media and compressed air. The differences, however, is that the wet blasting uses a third element, a liquid to enhance the surface finish [5]. The abrasive is suspended in a liquid projected at high velocity by a high pressured flow of compressed air [6]. The benefit of including water in the process is that it offers a much smoother and more consistent finish. 
In this experiment, the blasting installation uses as abrasive medium a corundum granulate and water as mixing medium. The concentration of abrasive in water is $15 \%$. The pressure of the compressed air is adjustable between 1 and 7 bar. Time is an important factor that determines the size of the cutting edge rounding. The distance between blasting nozzle and tool is $120 \mathrm{~mm}$. In order to obtain different sizes of the cutting edge rounding, the following blasting parameters were used:

- For the tool with the small cutting edge radius, an air pressure of 2 bar and 31 seconds as the total time of blasting was used.

- To obtain tools with a medium rounding, they were blasted for 130 second at 2 bar.

- The large cutting edge rounding was obtained by blasting the tools at 3.5 bar for 60 seconds.

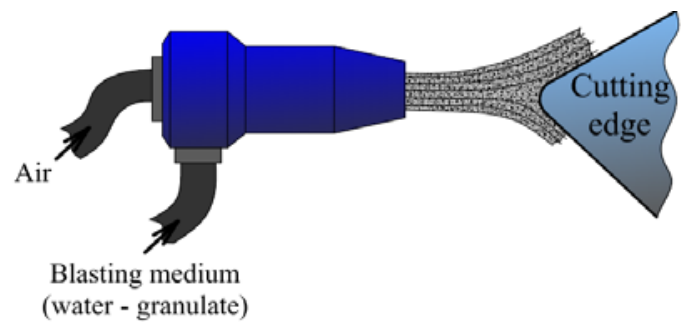

Fig. 4. Schematic representation of the cutting edge wet basting.

The tools cutting edge blasting process aims to increase the edge stability and rigidity, to eliminate the cutting edge defects and to smooth the edge surfaces.

In the following images, the uncoated tools with the cutting edge preparation are presented. From left to right the tool with a small cutting edge radius, the tool with a medium cutting edge radius and the tool with the large cutting edge radius.

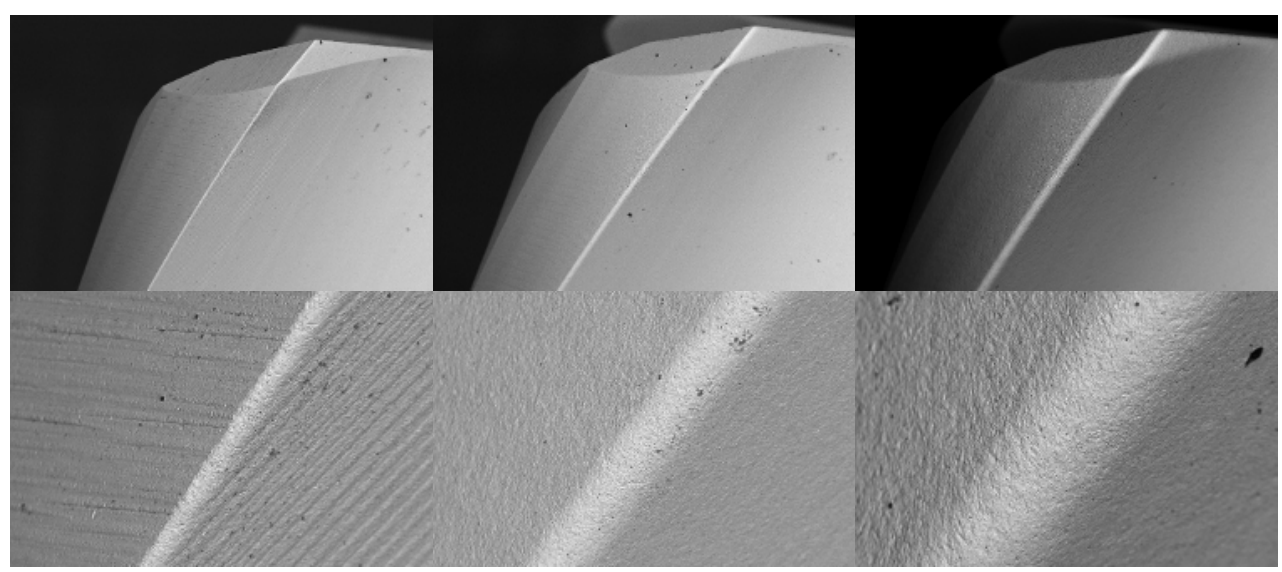

Fig. 5. Tools cutting edge after wet blasting.

\section{Coating}

After the wet blasting process the tools were coated with a multilayer coating of TiN and SiN which enhances the tool cutting performance. Guhring developed the new Signum coating that has a hardness of $5500 \mathrm{HV}$ one of the hardest nitride coatings on the market. Guhring could achieve this extraordinary coating hardness thanks to the special nano 
composite structure with a layer build-up of TiAlN and SiN. Thus, the Signum coating offers particularly high heat resistance and at the same time high diffusion resistance [7].

\section{Tool life test}

The durability tests were done on a 3 axis vertical CNC milling centre. The tools were analysed at predefined intervals to follow the edge wear and deterioration. At this intervals the wear was measured and the cutting edge was analysed at an electron microscope to see the edge wear, material adhesion and coating deterioration.

The intervals of documentation were done at first after $1 \mathrm{~m}$ and $3 \mathrm{~m}$ of cutting length. After that the documentation was done every $5 \mathrm{~m}$ until the maximum wear width of $360 \mu \mathrm{m}$ was achieved.

The cutting parameters were chosen after a functional cutting test through which the stability of the process was analysed. The following parameters were chosen: cutting speed $V_{c}=130 \mathrm{~m} / \mathrm{min}$ and the feed per teeth $f_{z}=0.03 \mathrm{~mm}$. The cutting depth $a_{p}$ is $12 \mathrm{~mm}$ and the cutting width $a_{e}$ is $2 \mathrm{~mm}$. These parameters are in the range of semi finishing cutting.

\section{Results and discussion}

The results of the durability tests showed that the tools with the small cutting edge radius show the greatest cutting performance with a $54.8 \%$ increase in tool life in comparison with the reference tool with the sharp edge. The tools with the medium and large cutting show a decrease in the tool life with $38.3 \%$ in the case of the tool with medium cutting edge rounding and with $72.7 \%$ in the case of the tool with small cutting edge rounding.

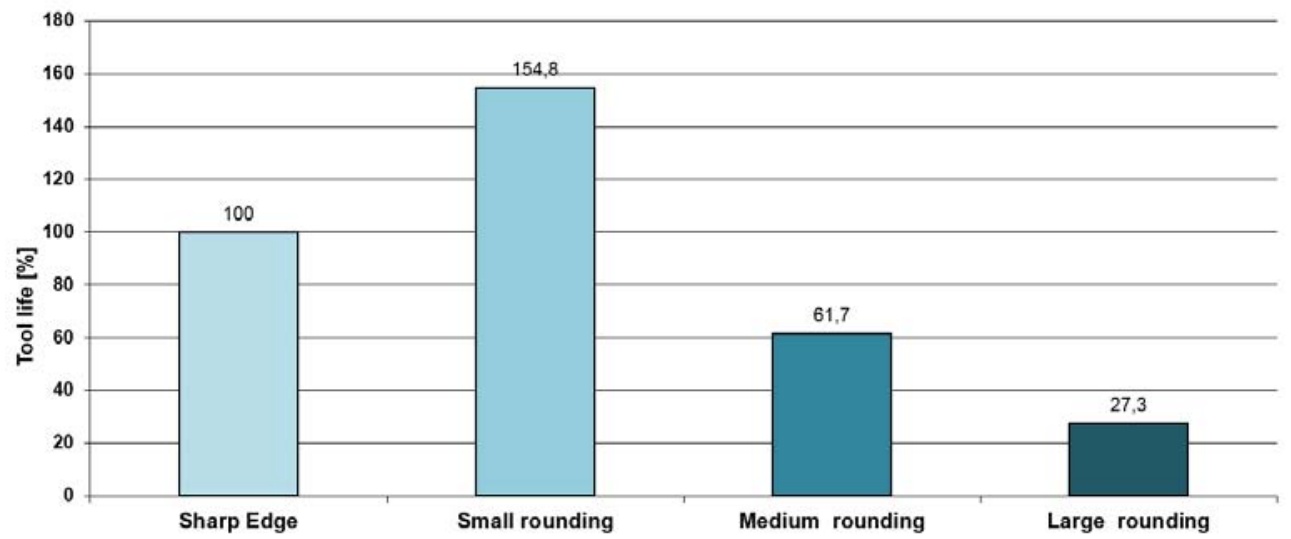

Fig. 6. Results of the tool life cutting tests

The tool with the sharp edge showed an excessive wear of the mill corner chamfer (Figure 7-a) which lead to an increase in tool vibration and a decrease of the surface quality of the machined workpiece and therefore the end of the cutting test.

In the case of the tool with the small cutting edge rounding (Figure 7-b) the wear is localised evenly alongside the cutting edge with a small increase on the mill corner chamfer. The surface quality of the machined workpiece was constant through all the tool life range and with no increase in the tool vibration. 
The wear of the tool with the medium and the big cutting edge radius (Figure 7-c and d) is distributed mostly on the cutting edge. This happened due to increased material deformation, increased temperature and material adhesion.

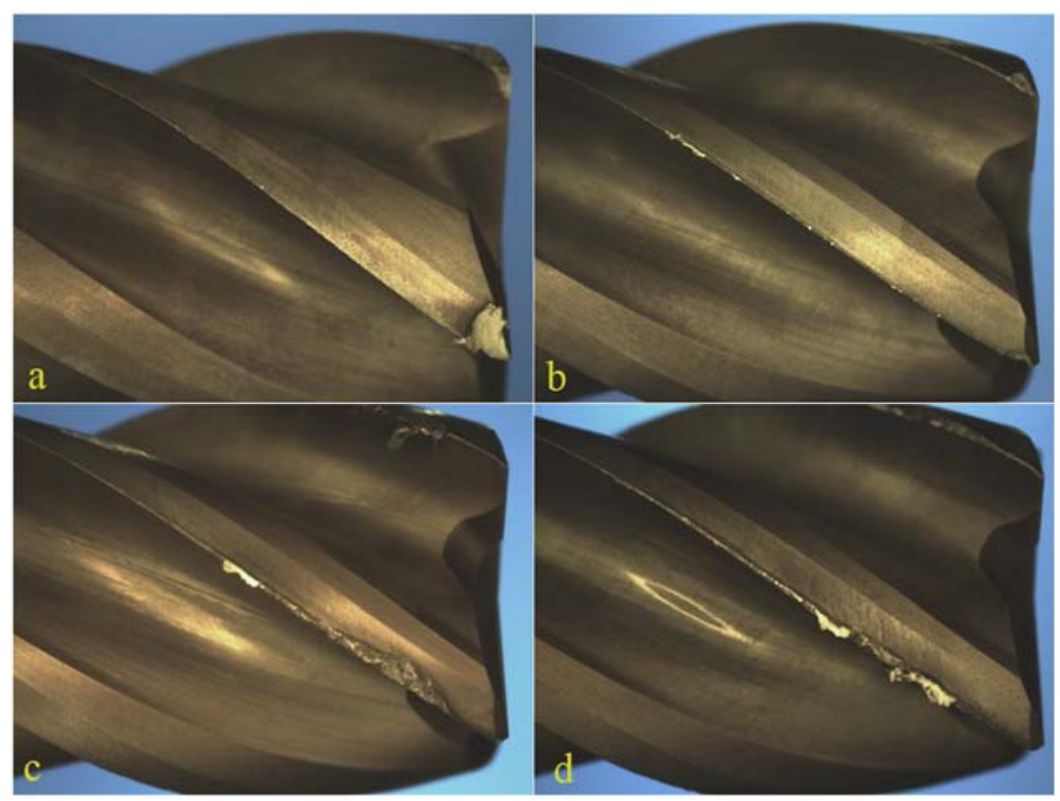

Fig. 7. The cutting edge wear at the end of the test.

\section{Conclusion}

It can be concluded that the Guhring RF100VA carbide end mill with a small cutting edge rounding that have been obtained by wet blasting the tool at 3.5 bar for 60 seconds using water and corundum as abrasive medium, have the best cutting performances and tool life in the process of machining a 1.4301 grade austenitic stainless steel.

The authors would like to thank the Gühring Company for the technical and material support and the Technical University of Cluj-Napoca for knowledge and information support.

\section{References}

1. H. Shao, L. Liu, H.L. Qu, Wear, 263, 736-744, (2007)

2. J.L. Endrino, G.S. Fox-Rabinovich, C. Gey, Surface \& Coatings Technology, 200, 6840-6845, (2006)

3. I. Ciftci, Tribology International, 39, 565-569, (2006)

4. B. Denkena, D. Biermann, CIRP Annals - Manufacturing Technology, 63, 631-653, (2014)

5. https://www.vapormatt.com/news/what-wet-blasting

6. http://www.azom.com/article.aspx?ArticleID=7894

7. http://pvdcoating.com.au/signum-coating/ 